\title{
SUL TEOREMA DI MALUS PEI RAGGI LUMINOSI CURVILINEI.
}

\author{
Nota di P. Pizzetti (Pisa).
}

Adunanza del 22 maggio 1910.

La interessante Nota del prof. H. Weber, contenuta nel fascicolo Maggio-Giugno I9 Io di questi Rendiconti ${ }^{2}$ ), la quale estende ai sistemi di raggi luminosi curvilinei il teorema di Malus, mi dà occasione di ricordare come nel 1890 io abbia data questa stessa generalizzazione ${ }^{2}$ ). Poichè il Periodico nel quale apparve quella mia Nota aveva pochissima diffusione, non posso lagnarmi che la Nota stessa sia rimasta quasi ignorata. Mi permetto ora di riprodurre qui la mia semplice dimostrazione, non per fare una questione di priorita, chè non ne varrebbe la pena, ma perchè quel mio modo di ragionare era una facile estensione della teoria delle ordinarie geodetiche, e questa estensione ha avuto al di d'oggi il massimo sviluppo colla teoria delle cosi dette linee estremali nel calcolo delle variazioni.

Assumiamo nello spazio un sistema di coordinate curvilinee $u, v, w$ e sia (I) $\quad d s^{2}=e . d u^{2}+2 f . d u \cdot d v+g . d v^{2}+2 h . d u . d w+2 k \cdot d v \cdot d w+l^{2} \cdot d w^{2}$ l'espressione del quadrato dell'elemento lineare. Posto

sarà

$$
\begin{gathered}
\frac{d u}{d w}=p, \quad \frac{d v}{d w}=q, \\
R=\sqrt{e p^{2}+2 f p q+g p q^{2}+2 h p+2 k q+l^{2}},
\end{gathered}
$$

$$
d s=R . d w
$$

e la condizione di minimo cui deve soddisfare ogni raggio luminoso potrd scriversi

$$
\int n R \cdot d w=\text { minimum, }
$$

ove con $n$ si denoti l'indice di rifrazione che si suppone variabile con continuità, e dove

I) H. Weber, Über den Satz von MaLus für krummlinige Lichtstrablen [Rendiconti del Circolo Matematico di Palermo, t. XXIX ( ${ }^{\circ}$ semestre I9Io), pp. 396-406].

2) P. Pizzetit, Sulle trajettorie dei raggi luminosi [Ateneo Ligure (Genova), vol. XIII (189o), pp. 202-225]. [Recensione: Jahrbuch über die Fortschritte der Mathematik, Bd. XXII (Jahrgang I89o), pp. 1067-1068]. 
l'integrale puó limitarsi fra due valori, entro certi limiti, arbitrarii della $w$. (Escludiamo il caso in cui il raggio luminoso giaccia sopra una superficie $w=$ cost., nel qual caso la $w$ non potrebbe naturalmente assumersi come variabile d'integrazione). Dalla (2) deduciamo al solito modo

$$
\frac{\partial}{\partial u}(n R)=\frac{d}{d w}\left(n \frac{\partial R}{\partial p}\right), \quad \frac{\partial}{\partial v}(n R)=\frac{d}{d w}\left(n \frac{\partial R}{\partial q}\right) .
$$

Supponiamo ora che le linee $w$ (ossia le linee lungo le quali sono costanti $u$ e $v$ ) siano raggi luminosi. Per tali linee si ha $R=l, \frac{\partial R}{\partial p}=\frac{b}{l}, \frac{\partial R}{\partial q}=\frac{k}{l}$, sicchè le (3) dànno

$$
\frac{\partial}{\partial u}(n l)=\frac{d}{d w}\left(\frac{n h}{l}\right), \quad \frac{\partial}{\partial v}(n l)=\frac{d}{d w}\left(\frac{n k}{l}\right) .
$$

Ora supponiamo che la famiglia delle superficie $w=$ cost. sia costituita come segue. Una di esse, per es. la $w=$ o che chiameremo $S_{0}$, sia una superficie regolare qualunque; da essa partano ortogonalmente i raggi luminosi $w$, i quali sono con ciò pienamente determinati. Sopra ciascuno di questi raggi si prenda, a partire da $S_{0}$ un arco $\sigma$ tale che l'integrale $\int n . d s$ esteso lungo $\sigma$ abbia un valore costante assegnato. Il luogo degli estremi di tali archi sarà una delle superficie $w=$ cost. Potremo allora porre $w=\int n . d s$, e sard quindi $l=\frac{\mathrm{I}}{n}$ e le (4) daranno $\frac{d}{d w}\left(n^{2} b\right)=0, \frac{d}{d w}\left(n^{2} k\right)=0$; ossia i prodotti $n^{2} b, n^{2} k$ conserveranno un valore costante lungo ciascuno dei raggi luminosi $q$. Escludiamo il caso che sia $n$ generalmente nullo. Allora, poichè per $w=0$ si ha $h=0, k=0$, qualunque siano $u$ e $v$ (giacchè le linee $w$ partono ortogonalmente da ogni punto della $S_{\mathrm{o}}$ ) saranno $b$ e $k$ nulli in tutto lo spazio. Le linee $w$ incontreranno dunque ortogonalmente tutte le superficie $w=$ cost. Con ciò è dimostrato che se una doppia infinità di raggi luminosi curvilinei taglia ortogonalmente una superficie, essa costituisce una congruenza normale. Dalla dimostrazione risulta poi che è costante l'integrale $\int n d s$ esteso lungo ogni linea della congruenza e compreso fra due superficie trasversali fisee.

Pisa, 18 maggio 1910.

P. PIzZETTI. 